\title{
Norois
}

Environnement, aménagement, société

242 | 2017

Espace fluvial, ancrage territorial, travail collaboratif, réseau

\section{Le lit endigué de la Loire moyenne : de l'image de fleuve sauvage à la reconnaissance de son caractère anthropisé}

The diked-up middle Loire river bed: from a wild river image to the recognition of its anthropogenic character

Francesca Di Pietro, Lotfi Mehdi, Clémence Chaudron et Fanny Moyon

\section{(2) OpenEdition}

Journals

Édition électronique

URL : http://journals.openedition.org/norois/6030

DOI : 10.4000/norois.6030

ISBN : 978-2-7535-5895-3

ISSN : $1760-8546$

Éditeur

Presses universitaires de Rennes

Édition imprimée

Date de publication : 22 juin 2017

Pagination : 7-23

ISBN : 978-2-7535-5893-9

ISSN : 0029-182X

\section{Référence électronique}

Francesca Di Pietro, Lotfi Mehdi, Clémence Chaudron et Fanny Moyon, « Le lit endigué de la Loire moyenne : de l'image de fleuve sauvage à la reconnaissance de son caractère anthropisé », Norois [En ligne], 242 | 2017, mis en ligne le 22 juin 2019, consulté le 03 janvier 2020. URL : http:// journals.openedition.org/norois/6030; DOI : 10.4000/norois.6030 


\pm Nöiels

\title{
Le lit endigué de la Loire moyenne : de l'image de fleuve sauvage à la reconnaissance de son caractère anthropisé
}

\author{
The Diked-up Middle Loire River Bed: From a Wild River Image \\ to the Recognition of its Anthropogenic Character
}

\author{
Francesca Di Pietro ${ }^{\mathrm{a} *}$, Lotfi Mehdi $^{\mathrm{b}}$, \\ Clémence Chaudron ${ }^{a}$ et Fanny Moyon ${ }^{c}$
}

\footnotetext{
*Auteur correspondant : tél : (33) 247361452

a UMR 7324 CITERES, Université François-Rabelais, Parc de Grandmont, 37200 Tours (France).

b UMR 7362 LIVE, Université de Strasbourg, 3 rue de l'Argonne, 67000 Strasbourg (France).

c Fish-Pass, 18 rue de la Plaine, Z.A. des 3 Prés, 35890 Laillé (France).
}

Résumé : Les espaces fluviaux font l'objet d'usages diversifiés et en évolution; cette diversité d'usages, sur des espaces relativement réduits, ne va pas sans conflit et sans poser de problèmes de gouvernance. La Loire a été investie, depuis une trentaine d’années, d'une valeur écologique et patrimoniale qui l'a identifiée comme un fleuve «sauvage », représentation liée à l'absence de grands ouvrages et au déclin des activités traditionnellement fluviales. Ce caractère «sauvage » de la Loire est plus particulièrement attaché à son lit endigué. L'objectif de ce travail est de proposer une démarche pour caractériser la diversité de l'occupation du sol du lit endigué de la Loire moyenne, à partir de la base de données du SIEL (Système d'Information sur l'Évolution du Lit de la Loire), seule base de données incluant les communautés herbacées. La diversité de l'espace inondable a été mise en relation avec ses dimensions longitudinale et transversale, en testant le lien entre l'occupation du sol, issue de descripteurs des communautés végétales, et des indicateurs de la distance amont/aval et de la largeur de l'espace fluvial. Nos résultats montrent que le gradient amont/aval est prépondérant, le lit endigué étant caractérisé par des forêts, prairies et sables à l'aval et par des surfaces artificialisées à l'amont. La largeur du lit endigué est corrélée avec la part de cultures, présentes en proportion non négligeable dans le lit endigué, et localisées de préférence là où le lit est le plus large. Nous mettons en évidence les usages que traduisent certains habitats de cet espace fluvial, qui ne se présente donc pas comme l'espace «sauvage » de certaines représentations sociales.

\begin{abstract}
Riparian landscapes show diversified and dynamic land uses; this diversity in relatively confined spaces involves conflicts and governance issues. Over the last thirty years, the river Loire has been vested with ecological and heritage values which have led to it being defined as a "Wild River". This perception is related to the lack of large dams and to the decline of traditional river activities. This "wild" aspect of the Loire is particularly linked to the diked-up river bed. This work aims to propose an approach to characterize the land cover diversity of the diked-up middle course of the Loire, based on the SIEL database (Information System on the Loire river bed Evolution) which takes into account herbaceous communities. The diversity of the flood area was linked to its longitudinal and cross-ways dimensions by analysing the relationship between land use, based on descriptors of plant communities, and indicators of the upstream/downstream distance and of the width of the river and its levees. Our results show that the upstream-downstream gradient dominates, the diked up river showing more forests, meadows and sand downstream and more impervious surfaces upstream. The width of the diked up river is correlated to the proportion of arable areas, a significant part of these being found on the river bed and localized particularly between the levees where the river is at its widest. Through studying the spatial diversity of the diked-up middle Loire, we have highlighted the anthropogenic practices reflected in some of the river's habitats, suggesting it is therefore not such a 'wild' space as depicted in current social representations.
\end{abstract}


Mots clés : paysage fluvial - occupation du sol - communautés végétales - usages de l'espace - nature

Keywords: river landscape - land cover - plant communities - land use - nature

\section{INTRODUCTION}

Les espaces fluviaux font l'objet d'usages diversifiés, croissants en ce qui concerne les activités récréatives, associées à une urbanisation en augmentation, et décroissants en ce qui concerne les activités d'élevage, associées aux prairies permanentes, en diminution (Harrison et al., 2010). Ces tendances sont communes aux fleuves des pays industrialisés (comme le montre le cas du Mid-West des États-Unis : Freeman et Ray, 2001). En France les fonctions récréatives grandissantes dévolues aux espaces fluviaux sont bien documentées pour un large panel de cours d'eau aux dimensions variées, et sont généralement en lien avec des espaces urbains en extension (Montembault, 2004; Romain, 2010; Ultsch, 2010 ; Féménias et Sirost, 2011 ; Romain, 2014). Le souci de mieux gérer l'emprise de l'urbanisation sur les corridors fluviaux, dans les zones inondables notamment, a conduit les pouvoirs publics à revaloriser la traversée urbaine des cours d'eau, qui constitue un espace visuel ou d'agrément. La Loire est ainsi largement perçue par les habitants des agglomérations tourangelle et orléanaise comme un élément de nature au sein de l'espace urbain (Rode, 2010b), et il a été remarqué que les aspects visuels priment sur ceux écologiques dans les projets urbains sur les fleuves (Bonin, 2007).

Dans le contexte de l'émergence, à l'échelle internationale, de nouveaux principes de gestion écologiques des cours d'eau s'opposant aux barrages (Barraud, 2011), la Loire a été investie, depuis une trentaine d'années, d'une valeur écologique et patrimoniale qui l'a identifiée comme un fleuve « sauvage»(Germaine et Barraud, 2013). Ceci est confirmé par une analyse des guides touristiques (Bonin, 2001), et par une analyse des représentations iconographiques dans le département du Maine-et-Loire, qui montre l'inflexion de la «naturalité » ligérienne, avec une sensibilité domestique au début du $\mathrm{xx}^{\mathrm{e}}$ siècle, évoluant aujourd'hui vers un registre «sauvage » (Joliet et al., 2004).
La représentation «sauvage » de la Loire est liée à l'absence de grands ouvrages, associés à de grandes installations industrielles, tels que ceux qui contraignent l'écoulement du Rhône, ainsi qu'au déclin des activités traditionnellement fluviales (pêche, navigation, pâturage) qui se traduit, dans plusieurs secteurs de Loire moyenne, par une « renaturalisation » du lit endigué (Bazin et Gautier, 1996). Ce caractère «sauvage » de la Loire est plus particulièrement attaché à son lit endigué (Folléa et Gautier, 2009), caractérisé en Loire moyenne par des bras multiples, séparés par des atterrissements sablonneux et des îles mises en place au cours du XIX ${ }^{\mathrm{e}}$ siècle (Grivel et Gautier, 2012).

Pourtant des travaux montrent que le lit de la Loire est un espace depuis longtemps structuré par les activités humaines, d'une part à travers la construction d'ouvrages submersibles, tels que les épis en Loire aval dès la fin du XVIII ${ }^{\mathrm{e}}$ siècle (Charrier, 2000) et au début du $\mathrm{xx}^{\mathrm{e}}$ siècle (Barraud et al., 2013), ou les aménagements de la Loire moyenne au XIX ${ }^{e}$ siècle (Lecœur, 2011). D'autre part des travaux interdisciplinaires montrent que les effets des facteurs anthropiques sur la dynamique fluviale sont lisibles dès le Néolithique moyen et surtout depuis l'époque gallo-romaine (Garcin et al., 2006) ; l'ancienneté de l'utilisation agricole de l'espace ligérien est attestée depuis le Néolithique, époque à laquelle prairies de fauche et pâturages mais aussi seigle, sarrasin, lin, céréales, étaient cultivés en Loire aval (Cyprien et al., 2004).

Le travail présenté ici porte sur la Loire moyenne, située entre le bec d'Allier et le bec de Maine, s'écoulant majoritairement dans un lit majeur endigué, c'est-à-dire bordé par des hautes levées de terre datant de plusieurs siècles, qui protègent une vaste plaine alluviale : les vals (Service de Bassin Loire-Bretagne, 2002). C'est sur l'espace endigué, ou « franc bord », c'est-à-dire la partie du lit majeur située entre les levées, qu'est centré le travail présenté dans cet article. 
Le premier objectif de ce travail est d'explorer la diversité de l'occupation du sol de l'espace endigué et de montrer qu'elle est en étroite relation avec des usages anthropiques intensifs.

Le deuxième objectif est de mettre en relation la diversité de l'occupation du sol du lit endigué avec des facteurs potentiellement explicatifs tels que ses dimensions longitudinale et transversale. La première impacte de façon générale l'organisation de l'espace fluvial (Pradeau, 1999), tandis que la seconde, liée aux modalités de l'endiguement, peut avoir un effet considérable sur l'occupation du sol, comme cela a été mis en évidence pour les plaines d'Europe occidentale (Van Looy et al., 2008). Pour ce faire nous testerons le lien entre l'occupation du sol et des indicateurs de la distance amont/aval (dimension longitudinale) et de la largeur (dimension transversale) de l'espace fluvial.

Sur le plan méthodologique nous proposons une démarche pour caractériser la diversité de l'occupation du sol du lit endigué de la Loire, basée sur l'utilisation d'une base de données construite et mise à disposition par les services de l'état (DREAL), et impliquant un reclassement de descripteurs hétérogènes pour aboutir à une caractérisation de l'occupation du sol.

\section{Site D'ÉTUde ET MÉTHOdologie}

\section{Le site d'étude : la Loire moyenne endi- guée d'Orléans à Montsoreau}

L'espace étudié est délimité par les communes d'Orléans (Loiret), à l'amont, et de Montsoreau (Maine-et-Loire), à l'aval (figure 1), ce qui nous permet de nous centrer sur le Val de Loire et de disposer de données d'occupation du sol homogènes. Ce site d'étude, qui traverse 76 communes, a une longueur de 171,19 km.

\section{Les données du Système d'Information sur l'Évolution du Lit de la Loire (SIEL)}

Afin d'analyser un espace largement occupé par des surfaces herbacées peu intégrées dans les systèmes de production agricole et dans les réseaux

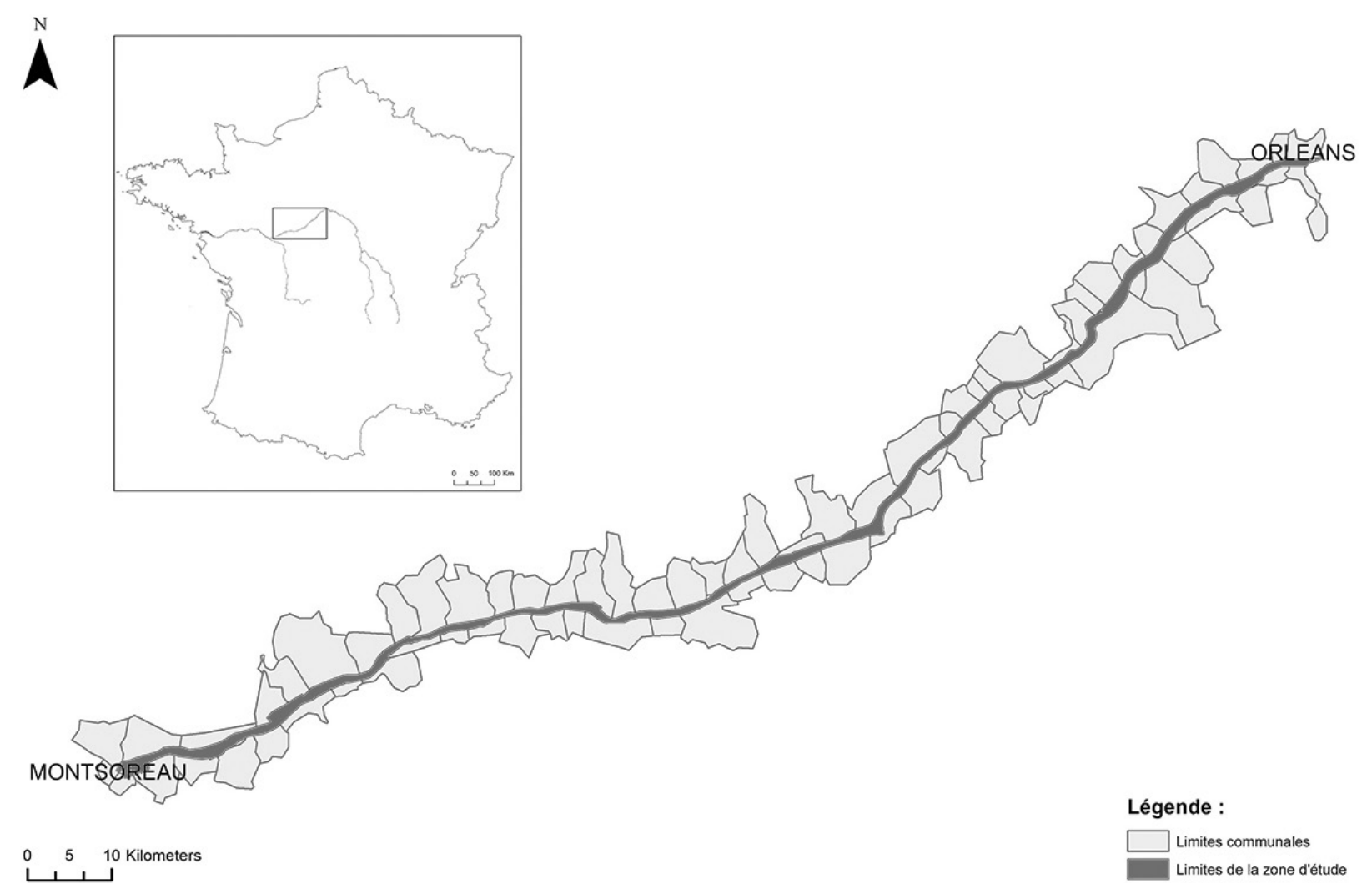

Figure 1: Le site d'étude : le lit endigué de la Loire moyenne d'Orléans à Montsoreau The study site : the diked-up middle Loire river bed from Orléans to Montsoreau 


\begin{tabular}{|l|l|}
\hline \multicolumn{2}{|l|}{ Catégories de rang 1 (SIEL) } \\
\hline 1 & Hydrographie \\
\hline 2 & Végétation aquatique et semi-aquatique \\
\hline 3 & Végétation herbacée pionnière typique du lit mineur \\
\hline 4 & Végétation herbacée, lande et friches à l'écart du lit mineur \\
\hline 5 & Forêts alluviales \\
\hline 6 & Autres forêts et boisements \\
\hline 7 & Cultures \\
\hline 8 & Espaces anthropisés \\
\hline
\end{tabular}

Tableau 1 : Premier rang de la typologie du SIEL (à gauche) et classification utilisée, issue du regroupement des classes des polygones (à droite) First rank typology of Loire river bed (on the left), and used classification, derived from polygone's classes aggregation (on the wright)

\begin{tabular}{|l|l|}
\hline \multicolumn{2}{|l|}{ Classification utilisée } \\
\hline 1 & Sables \\
\hline 2 & Eau courante et stagnante \\
\hline 3 & Végétation aquatique et semi-aquatique \\
\hline 4 & Végétation pionnière du lit mineur \\
\hline 5 & Prairies du lit majeur \\
\hline 6 & Pelouses et friches \\
\hline 7 & Forêts de bois tendre \\
\hline 8 & Autres forêts \\
\hline 9 & Cultures \\
\hline 10 & Vignes et vergers \\
\hline 11 & Jachères \\
\hline 12 & Espace artificialisé \\
\hline
\end{tabular}

urbains, nous avons utilisé les données du Système d'Information sur l'Évolution du Lit de la Loire (SIEL). Inspiré par le Plan Loire Grandeur Nature (1994) et initié sur la Loire moyenne, le SIEL est une base de données élaborée et mise à disposition du public par la DREAL Centre-Val de Loire ${ }^{1}$. Les données les plus récentes disponibles en Loire moyenne datent de 2005 ; la mission de 2005, qui se termine à l'aval de la commune de Montsoreau (49), est aussi la seule mission continue de Loire moyenne, pour laquelle il n'y a pas de discontinuités spatiales, ce qui n'est pas le cas pour les missions précédentes.

\section{Des classes de végétation aux classes d'occupation du sol}

Les cartes de végétation du SIEL, élaborées à partir de l'interprétation de photos aériennes prises à l'étiage et validées par une visite de terrain, décrivent la végétation dominante du lit mineur de la Loire, sur la base de la physionomie de la végétation, plus que des groupements végétaux (DIREN CENTRE, 1994). Les surfaces sont déterminées en fonction principalement de groupements-types définis dans la typologie simplifiée des communautés végétales du lit endigué de la Loire (Cornier, 2002), une typologie fondée sur les espèces dominantes et sur un ou plusieurs gradients écologiques. Cette typologie

1. [http://www.centre.developpement-durable.gouv.fr/les-cartes-de-vegetationr574.html]. est structurée en quatre rangs et comporte huit catégories de rang 1 : à des classes de végétation sont ajoutées des grandes classes d'occupation du sol non détaillées, de façon à couvrir de façon continue la totalité de l'espace endigué.

Dans le SIEL notre site d'étude est constitué de 16706 polygones, affecté chacun à une catégorie de végétation ou d'occupation du sol; la précision relative des images est inférieure à $5 \mathrm{~m}$. Les 16706 polygones dans leur ensemble sont affectés à 68 catégories appartenant à différents rangs de la typologie : excepté pour les forêts alluviales, la majorité des catégories se révèlent être de rangs 3, 2 et 1 , ce dernier, le moins précis, étant la seule affectation commune à tous les polygones. Les huit catégories de rang 1 étant insuffisamment détaillées, nous avons regroupé les 68 catégories présentes sur le site d'étude en 12 classes, qui s'apparentent à des types d'occupation du sol (tableau 1). Par rapport à la classification du SIEL de rang 1 nous avons notamment isolé les sables (une particularité de l'hydrosystème ligérien), séparé les prairies des pelouses (habitats herbacés bien différents), isolé les forêts de bois tendres, liées à l'intensité et à la fréquence de l'inondation; nous avons aussi séparé les cultures en trois classes, isolant notamment les cultures permanentes (vergers et vignes) et les jachères. 


\section{Segmentation du site d'étude}

Afin d'explorer la diversité amont-aval de ce site, nous avons découpé cet espace en tronçons d'égale longueur, à travers un traitement géomatique effectué avec le logiciel ARCGIS 10.1 et synthétisé dans la figure 2 (planche I). Trois étapes ont été suivies :

- tous les polygones représentant les communautés végétales, soit 16706 polygones (figure 2-1 planche I), ont été fusionnés en un seul polygone (figure 2-2 - planche I), qui représente l'ensemble de notre site d'étude : la Loire moyenne endiguée d'Orléans à Montsoreau;

- afin de calculer la longueur de ce secteur, nous avons tracé une ligne médiane (avec la fonction : Collapse Dual Lines To Centerline), après avoir transformé ce polygone en polylignes (fonction Polygon To Line) (figure 2-3 - planche I);

- la médiane a été découpée (fonction Split) en dix segments d'égale longueur, par une ligne perpendiculaire, de façon à obtenir dix polygones, que nous appellerons «tronçons », d'une longueur de 17,1 km chacun (figure 2-4 - planche I).

Les tronçons ainsi définis ne sont pas morphologiquement homogènes et n'incluent pas les versants, comme c'est le cas dans d'autres travaux sur les paysages de vallée (Germaine, 2008). Ils ne sont pas non plus caractérisés par une homogénéité d'occupation du sol : il s'agit de tronçons d'égale longueur créés pour explorer la diversité de l'occupation du sol et de la largeur du lit endigué.

\section{Calcul de la largeur des tronçons et de leur place dans le gradient amont-aval}

Afin d'obtenir un indicateur de la largeur des tronçons, nous avons tracé la droite perpendiculaire au centroïde de chaque tronçon, qui définit un segment que nous appellerons largeur médiane du tronçon; la figure 3 (planche II) présente l'exemple du tronçon $n^{\circ} 7$. La distance des centroïdes à l'extrémité amont du site d'étude a été calculée de façon à disposer d'un indicateur de la position de chaque tronçon dans la dimension longitudinale du fleuve.

\section{Analyses effectuées}

Afin d'explorer la diversité de l'occupation du sol des tronçons nous avons utilisé une classification ascendante hiérarchique (CAH) des tronçons (corrélations de Spearman entre les pourcentages de la surface du tronçon dans les différentes classes d'occupation du sol), ainsi que des analyses multivariées (Analyse en Composantes Principales sur ces mêmes pourcentages).

Pour étudier le lien entre l'occupation du sol d'une part, et la largeur médiane des tronçons et leur distance à l'extrémité amont du site, d'autre part, nous avons utilisé des analyses sous-contrainte et notamment une analyse de redondance (Redundancy Detrended Analysis : RDA) qui permet de mesurer la contribution d'une ou plusieurs variables (largeur et distance à l'amont) à la variance d'une matrice descriptive (les classes d'occupation du sol). Il s'agit là d'analyses communément utilisées en écologie du paysage et des communautés (ter Braak et Wiertz, 1994 ; Leps et Smilauer, 2003).

Le test de corrélation de Spearman, test nonparamétrique plus adapté que celui de Pearson aux échantillons de petite taille, a été utilisé pour mesurer le lien entre deux variables quantitatives continues.

Les logiciels utilisés sont CANOCO5 (ACP, RDA) et XLSTAT (CAH, test de Spearman).

\section{RÉSUltats}

\section{Dix tronçons hétérogènes}

Les résultats de la segmentation montrent dix tronçons à la largeur très variable : entre 700 et $1300 \mathrm{~m}$ environ (figure 4 - planche III et tableau 2).

\section{Diversité de l'occupation du sol du lit endigué de la Loire moyenne}

À l'échelle de l'ensemble du site d'étude la classe d'occupation du sol la plus représentée est Eau courante et stagnante, suivie par les Prairies du lit majeur, les forêts (Forêts de bois tendre et Autres forêts), les Cultures et l'Espace artificialisé; les classes les moins représentées sont les Sables, la Végétation aquatique et semi-aquatique, la Végétation pionnière du lit mineur, les Pelouses et friches, les Vignes et vergers 


\begin{tabular}{|c|c|c|c|c|}
\hline \multirow{2}{*}{$N^{\circ}$ tronçon } & commune est & commune ouest & largeur médiane $(\mathrm{m})$ & $\begin{array}{c}\text { distance à l'extrémité } \\
\text { amont }(\mathrm{km})\end{array}$ \\
\hline 1 & Orléans & Mareau-aux-Prés & 1144,99 & 8,56 \\
\hline 2 & Mareau-aux-Prés & Saint-Laurent-Nouan & 1334,27 & 25,68 \\
\hline 3 & Saint-Laurent-Nouan & Cour-sur-Loire & 962,57 & 42,80 \\
\hline 4 & Cour-sur-Loire & Chouzy-sur-Cisse & 730,35 & 79,92 \\
\hline 5 & Chouzy-sur-Cisse & Mosnes & 670,08 & 94,15 \\
\hline 6 & Mosnes & Noizay & 897,79 & 111,27 \\
\hline 7 & Noizay & Tours & 965,95 & 128,39 \\
\hline 8 & Tours & Villandry & 669,58 & 145,51 \\
\hline 9 & Villandry & Saint-Patrice & 1233,78 & 162,63 \\
\hline
\end{tabular}

Tableau 2 : Limites communales, largeur médiane et distance à l'amont des tronçons étudiés Municipal boundaries, median width and distance to upstream of the studied sections

et les Jachères (figure 5). Nous observons que les cultures (classe 9) sont la classe qui présente la plus grande variabilité en ce qui concerne la contribution à l'occupation du sol des tronçons.

À l'échelle des tronçons l'occupation du sol du lit endigué de la Loire moyenne montre, de l'amont (tronçon 1) à l'aval (tronçon 10), une proportion relativement croissante de forêts (classes 7 et 8 ) et de sables, et une proportion relativement décrois- sante de cultures, de vignes et vergers et d'espace artificialisé (figure 6 - planche IV).

Les analyses suivantes confirment la structuration longitudinale de la diversité de l'occupation du sol du lit endigué.

Une classification ascendante hiérarchique $(\mathrm{CAH})$ des tronçons étudiés, effectuée en mesurant les corrélations de Spearman entre les classes d'occupation du sol, montre que la variabilité de l'occupation du

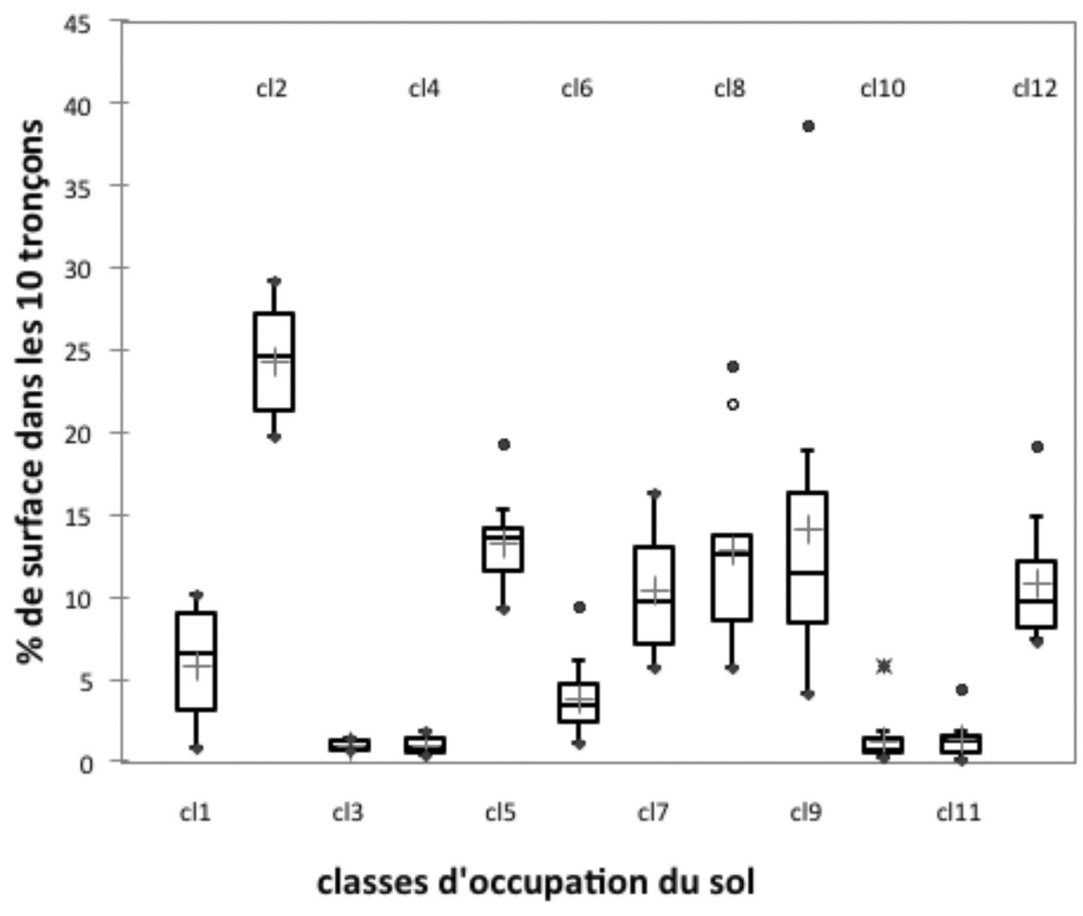

+ Moyenne ${ }^{\circ}$ Valeurs extrêmes(1) * Valeurs extrêmes(2) • Minimum/Maximum
Figure 5 : Variabilité de l'occupation du sol du site d'étude

En ordonnée : les valeurs mesurées dans les tronçons étudiés. En abscisse : les classes d'occupation du sol (cl) (rappel : 1 : Sables; 2 : Eau courante et stagnante; 3 : Végétation (semi)aquatique; 4 : Végétation pionnière du lit mineur; 5 : Prairies du lit majeur; $6: \mathrm{Pe}-$ louses et friches; 7 : Forêts de bois tendre; 8 : Autres forêts; 9 : Cultures; 10 : Vignes et vergers; 11 : Jachères; 12 : Espace artificialisé). study site Variability of the land cover in the

On the vertical axis, the values measured in the studied sections. On the horizontal axis the classes of land cover (cl), listed in table 2. 

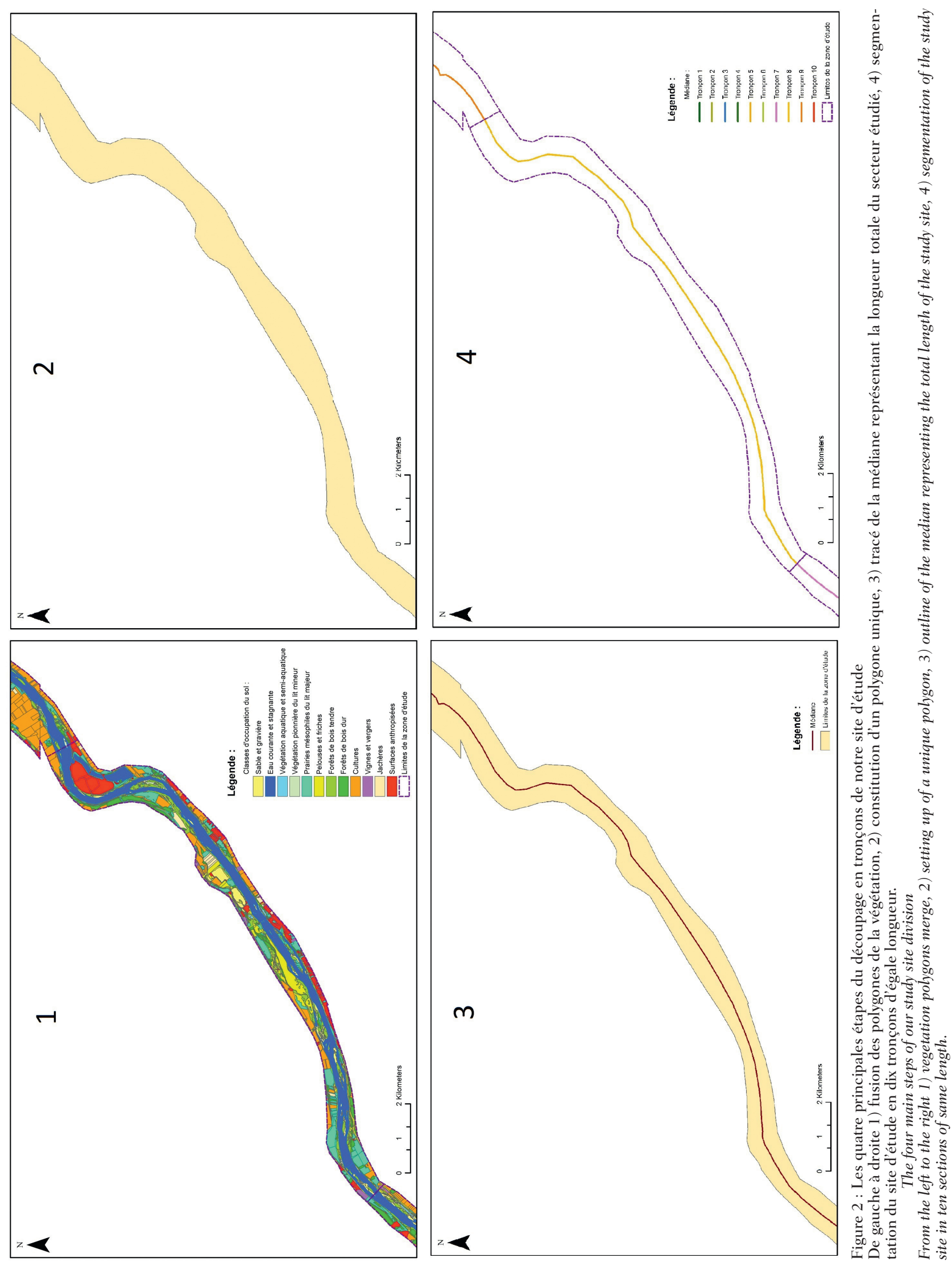

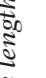


Planche II (Francesca Di PIETRO et al. - Le lit endigué de la Loire moyenne...)

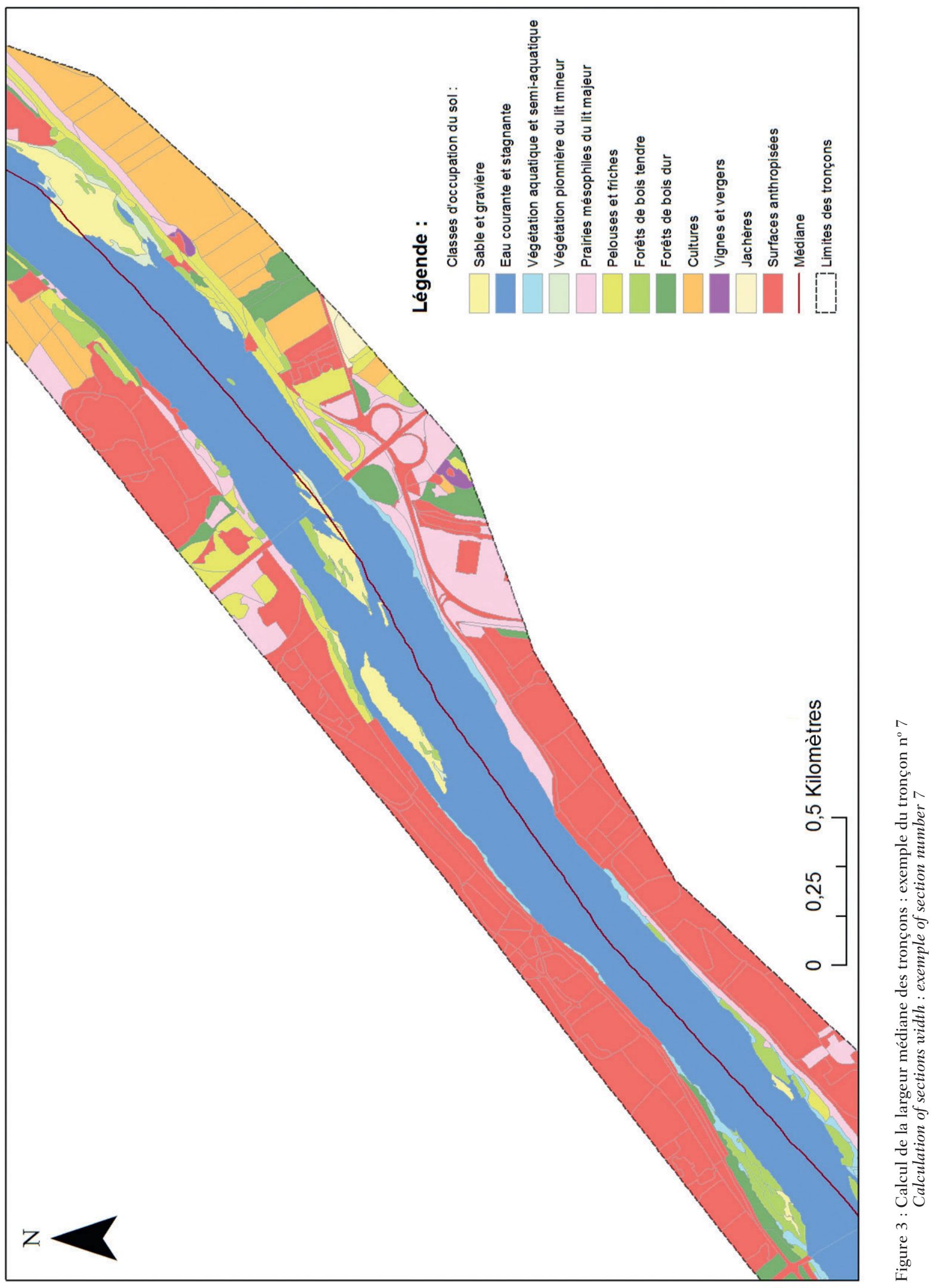




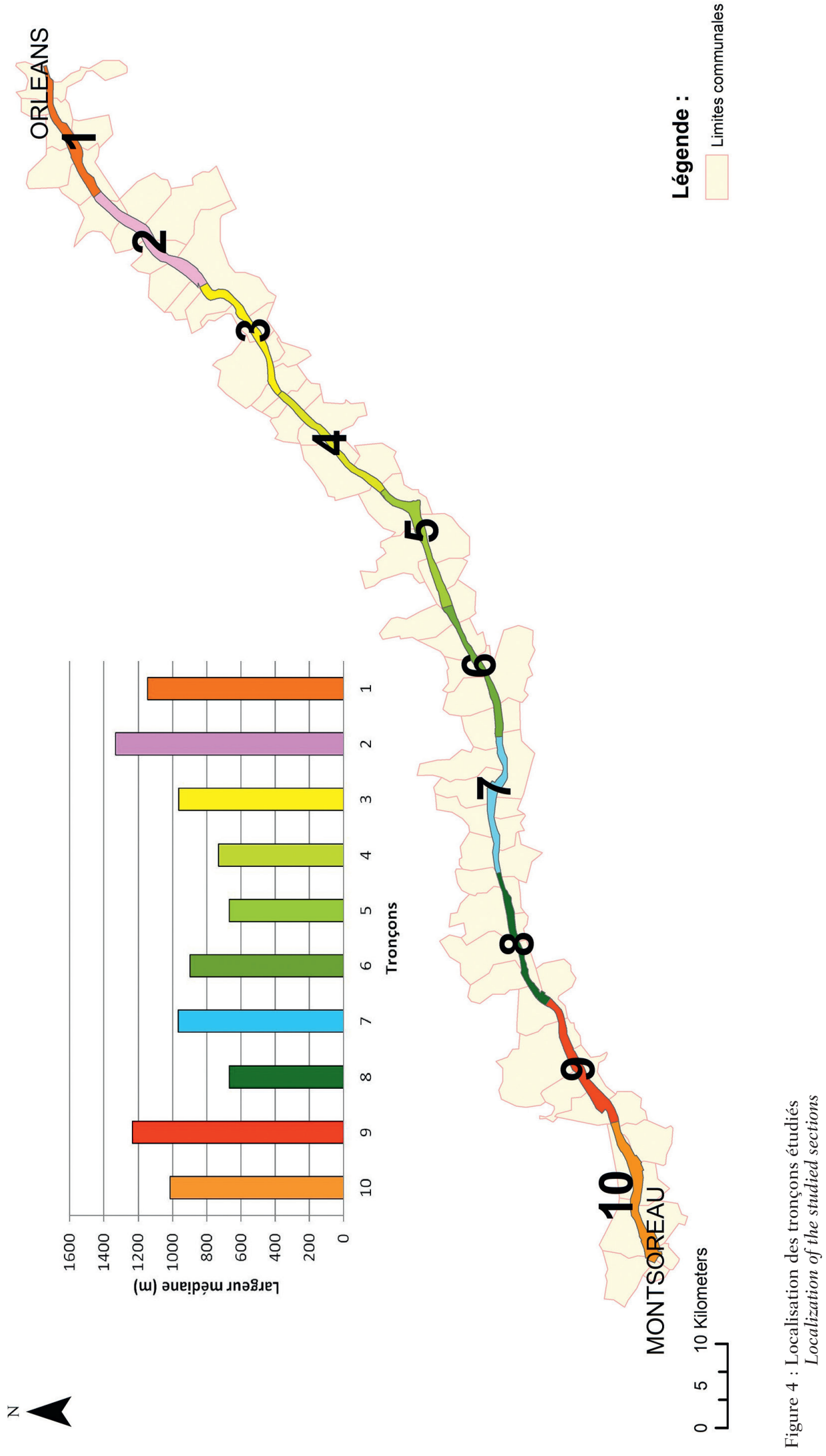




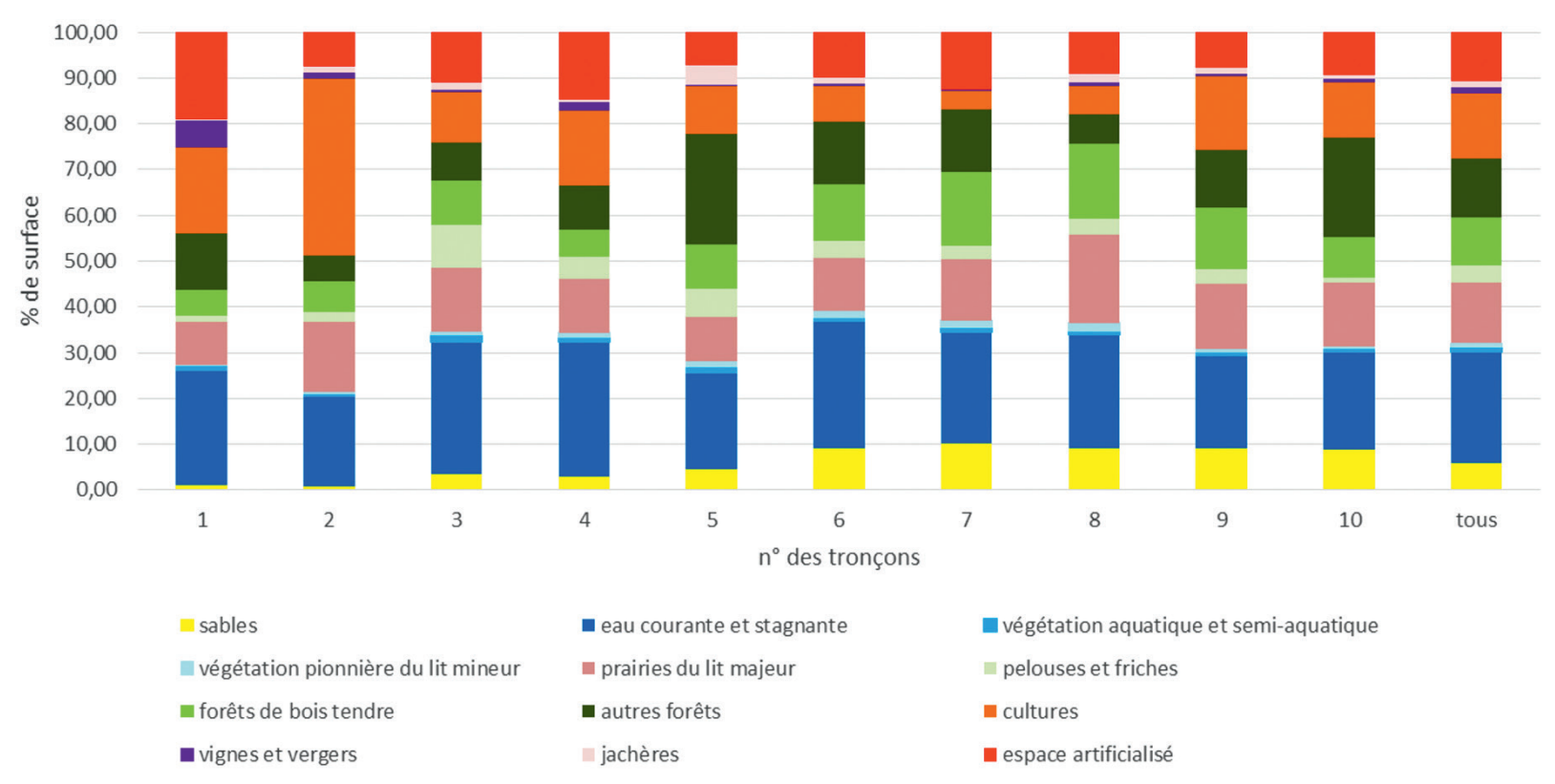

Figure 6 : Part de chaque classe d'occupation du sol dans la surface des tronçons du site d'étude Share of each class of land cover in the study site sections 
sol dans les tronçons peut être synthétisée en trois types de tronçons :

- les tronçons de l'amont ( $n^{\circ} 1$ à 4), qui ont la plus forte proportion de cultures (21\% de la surface en moyenne) et d'espace artificialisé et la plus faible proportion de sables et de forêts (tout type confondu);

- les tronçons $\mathrm{n}^{\circ} 6,7,8$, qui ont la plus forte proportion de forêts de bois tendres (15\% de la surface en moyenne) et de sables (9\%) et la plus faible proportion de cultures;

- les tronçons $\mathrm{n}^{\circ}$ 5, 9, 10, qui ont la plus forte proportion de forêts de bois durs et autres $(20 \%$ en moyenne) et la plus faible proportion d'espace artificialisé.

Le premier axe de l'Analyse en Composantes Principales (figure 7), corrélé avec la distance à l'amont (Spearman $=0,673, \mathrm{p}=0,037$ ), oppose la part de cultures et de vignes et vergers, liée aux tronçons de l'amont ( $n^{\circ} 1$ et 2$)$ à la proportion de sables, de végétation herbacée (végétation pionnière du lit mineur, classe 4 , et prairies du lit majeur, classe 5) et de forêt (classes 7 et 8), liée aux tronçons de l'aval $\left(n^{\circ} 6,8,9\right)$.

Le deuxième axe de l'ACP, négativement corrélé avec la largeur médiane des tronçons (Spearman = $-0,733, \mathrm{p}=0,01)$, isole les proportions de jachères et de pelouses et friches (liées aux tronçons $n^{\circ} 5$ et 3$)$.

\section{Lien avec la dimension de l'espace inter-digues et le gradient amont/aval}

Les dimensions longitudinale et transversale du site d'étude ne sont pas corrélées entre elles, la largeur médiane des tronçons n'étant pas proportionnelle à la distance à l'amont sur cette partie de la Loire. La largeur du lit endigué est en revanche corrélée avec la part de cultures dans les tronçons (Spearman $=0,685, \mathrm{p}=0,033)$, tandis que la distance à l'amont est corrélée avec la part de sables (Spearman $=0,745, \mathrm{p}=0,017)$.

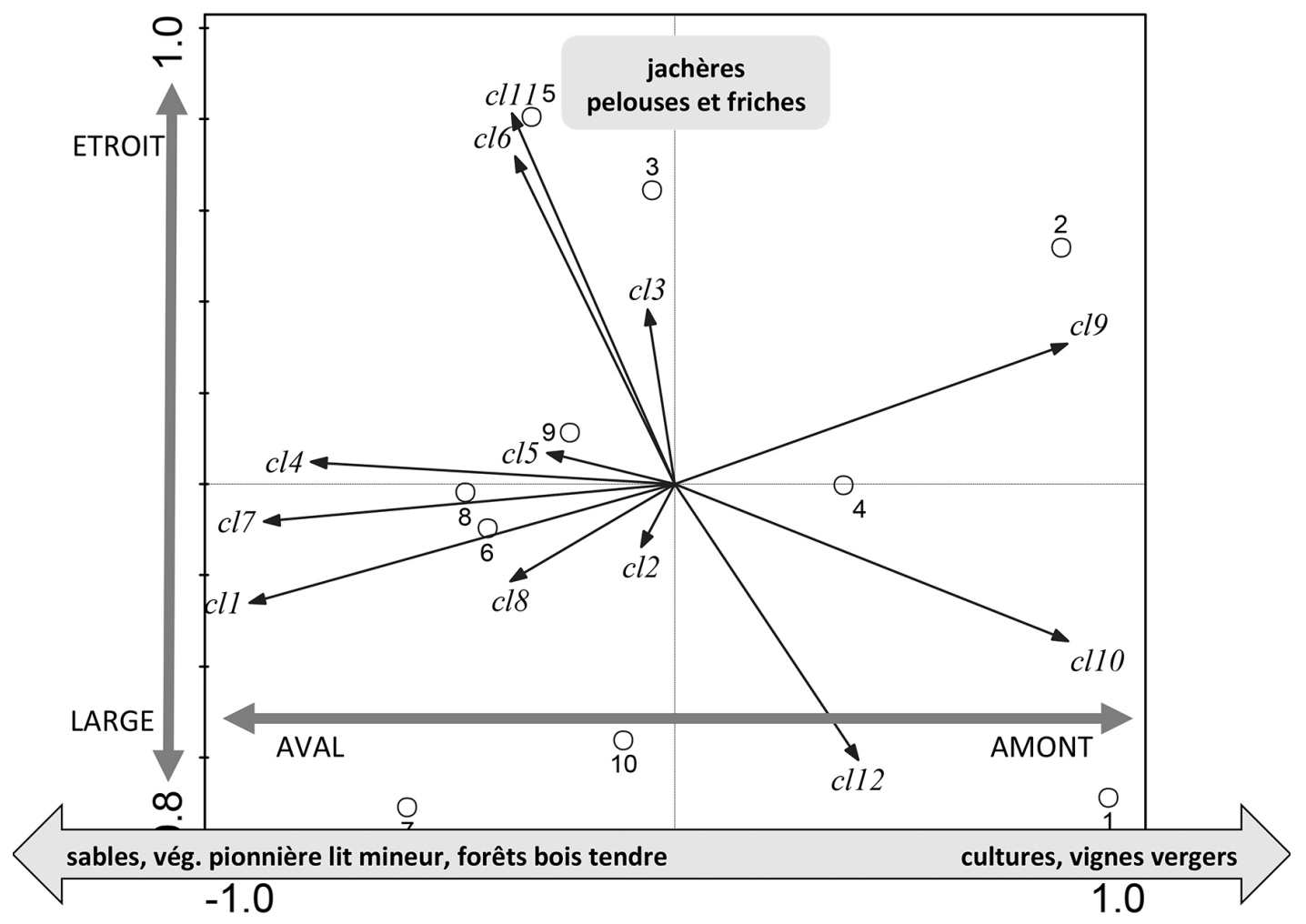

Figure 7 : Principal plan de l'Analyse en Composantes Principales sur les pourcentages des classes d'occupation du sol (représentées par des flèche) dans les tronçons (représentés par des cercles); en abscisse l'axe 1, en ordonnée l'axe 2

Main plan of Principal Component Analysis on the percentages of land cover classes (represented by arrows) in the sections (represented by circles); abscissa axis 1, on the ordinate axis 2 
Les deux premiers axes de l'analyse de redondance (RDA) expliquent 56,35\% de la variance de l'occupation du sol (figure 8); le gradient amont/aval est prépondérant et lié à l'axe principal de la RDA. La distance à l'amont est directement liée à la part de forêts (classes 7 et 8 ), de jachères, de prairies du lit majeur, de sables (tronçons aval: $\mathrm{n}^{\circ} 10,8,9$ ) et inversement liée à la part d'espace artificialisé et de vergers et vignes (tronçons amont: $\mathrm{n}^{0} 1,2,4$ ). Vers l'aval il y a donc plus de forêts, de prairies et de sables, tandis que vers l'amont il y a plus d'espace artificialisé.

Parmi les deux paramètres explicatifs de la diversité de l'occupation du sol du lit endigué mesurés, la largeur médiane et le gradient amont-aval, c'est donc ce dernier qui est le plus explicatif, bien que la proportion de surface de la classe majoritaire, l'eau courante et stagnante $(24 \%$ de la surface en moyenne), soit inversement liée à la largeur du lit endigué (logiquement l'eau est dominante lorsque le lit est étroit; inversement les cultures dominent là où le lit est large). La largeur du lit endigué est directement liée à la part de cultures et inversement liée à la part de pelouses et friches.

\section{DES HABITATS DIVERSEMENT LIÉS À L'EAU}

Comme attendu, nous observons donc que le lit endigué de la Loire moyenne en aval d'Orléans comprend des habitats semi-naturels comme les prairies du lit majeur qui sont, du point de vue écologique, la classe la plus hétérogène de la typologie du SIEL, comprenant ici des prairies humides, prairies mésophiles et mésoxérophiles. Dans leur diversité ces habitats sont souvent en lien avec les activités de pâturage, passées ou en usage, ou, pour les moins humides, de fauche (Cornier, 2002); ici ces habitats sont présents de façon relativement régulière dans tous les tronçons; sur la Meuse ces habitats sont structurés en communautés de plus en plus isolées (Van Looy et Meire, 2009). C'est sur ce type d'habitat que repose l'image de la Loire comme fleuve sauvage. Or, dans la Loire moyenne, étudiée ici, les prairies du lit majeur ne représentent que $13 \%$ de la surface en moyenne (entre $9 \%$ et $19 \%$ de la surface des tronçons).

Nos résultats montrent que le lit endigué de la Loire moyenne comprend des habitats très anthro-

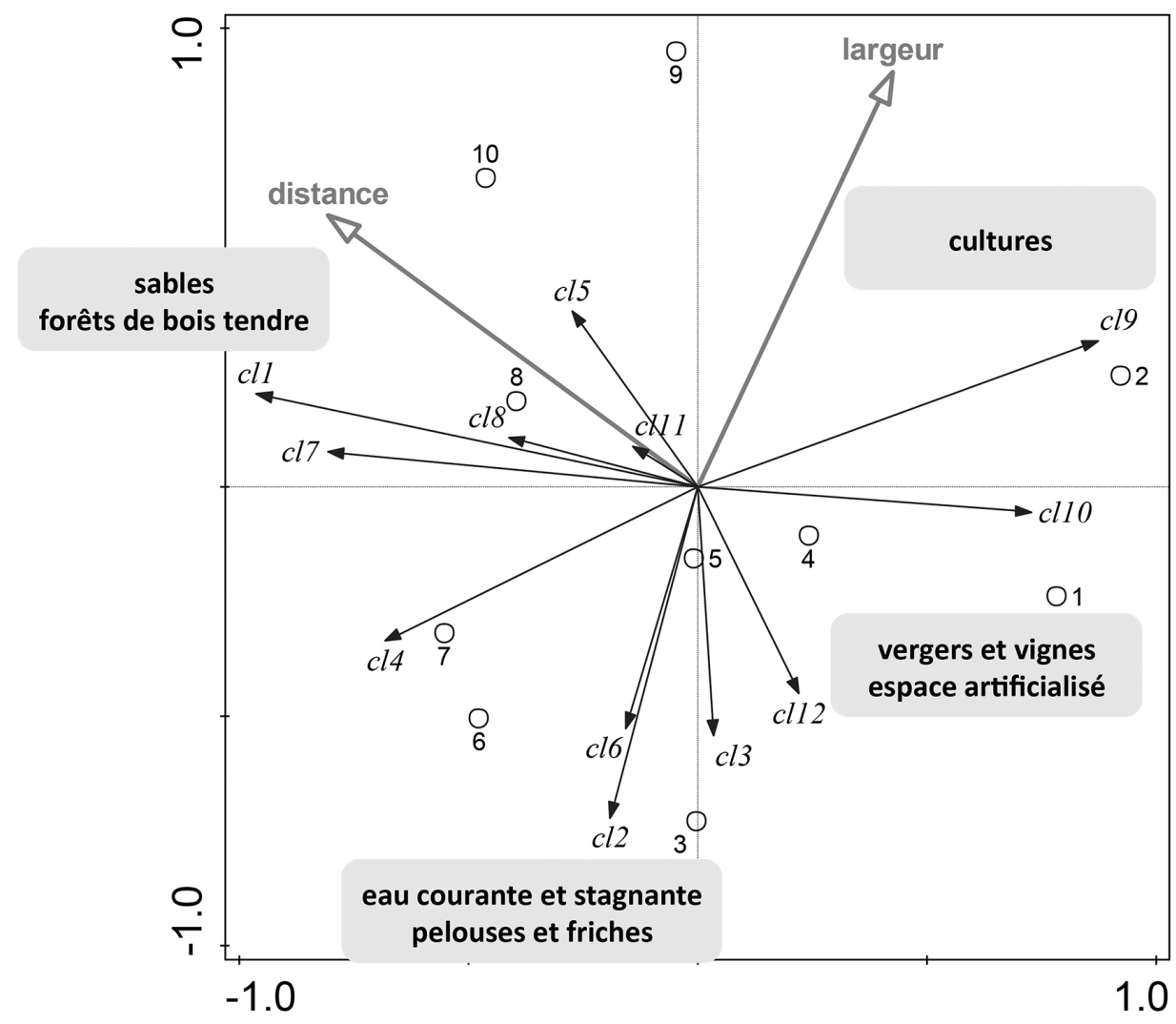

Figure 8 : Principal plan de l'analyse de redondance sur les classes d'occupation du sol (représentées par des flèches noires) dans les tronçons (représentés par des cercles)

Les variables explicatives, soit la largeur médiane des tronçons et la distance à l'amont, sont représentées par les flèches grises; en abscisse l'axe 1, en ordonnée l'axe 2 .

Main plan of the Redundancy Detrended Analysis on the land cover classes (represented by black arrows) in the sections (represented by circles) The explanatory variables, ie the sections median width and the distance to the upstream, are represented by grey arrows; abscissa axis 1 , on the ordinate axis 2. 
pisés et non compatibles avec les inondations : cultures, espaces artificialisés, forêts autres que celles de bois tendre. L'ensemble des cultures et des espaces artificialisés, que la typologie du SIEL, orientée vers les communautés végétales, ne détaille pas, représentent ainsi entre $15 \%$ (tronçon $n^{\circ} 8$ ) et $46 \%$ (tronçon $\mathrm{n}^{\circ} 2$ ) de la surface du lit endigué.

Les cultures constituent $14 \%$ de la surface; cette étude montre en effet que le lit de la Loire moyenne est en partie cultivé. Dans le site étudié il s'agit de l'occupation de sol à l'emprise la plus variable entre les tronçons, tout en étant plus élevée à l'amont. L'intensification agricole des plaines d'inondation, étudiée pour d'autres fleuves comme l'Isère (Girel, 2010), la Meuse (Van Looy et Meire, 2009) ou le Haut-Rhône (Pautou et Bravard, 1982), est particulièrement contrastée pour la Loire et ses affluents, à la fois dans les usages (Gueydon et Maillet, 2010; Gatien et al., 2011) et dans les représentations de l'espace fluvial. Des travaux montrent une extension progressive des cultures intensives dans le lit endigué de la Loire moyenne, en relation avec l'incision du lit, dès le milieu du XIX ${ }^{\mathrm{e}}$ siècle (Bazin et Gautier, 1996) alors que l'opposition entre le val intensivement cultivé et le lit endigué délaissé par les activités pastorales se manifeste au $\mathrm{Xx}^{\mathrm{e}}$ siècle (Burnouf et al., 2005).

L'espace artificialisé dans son ensemble est constitué de surfaces imperméabilisées, comme les emprises routières et les zones bâties; il représente $11 \%$ du lit en Loire moyenne, de façon relativement régulière entre les tronçons, avec une emprise considérable dans les tronçons $n^{\circ} 1$ (agglomération d'Orléans), $n^{\circ} 4$ (Blois) et $n^{\circ} 7$ (Tours). L'artificialisation croissante des cours d'eau est liée d'une part à la lutte contre les crues, en particulier pour les petites rivières péri-urbaines (Rollin, 2010), d'autre part à l'utilisation des berges pour la construction de quais, qui ont été accaparés par la circulation automobile ou transformés en parkings (Pelletier, 1990).

Les forêts autres que la forêt de bois tendre représentent presque $20 \%$ de la surface du site d'étude (la forêt de bois tendre représentant $10 \%$ de la surface), et ont une emprise hétérogène dans les tronçons (elles concernent particulièrement les tronçons $\mathrm{n}^{\circ} 5$ et 10). L'extension de la forêt dans l'espace fluvial a été attestée en Loire amont depuis le XVIII ${ }^{\mathrm{e}}$ siècle (Leteinturier et al., 2000), sur le Rhône à partir de la seconde moitié du XIX ${ }^{\mathrm{e}}$ siècle (Fruget et Dessaix, 2003); dans le Haut-Rhône l'évolution vers les biocénoses terrestres conséquente à l'endiguement et au drainage a été également soulignée (Pautou et Bravard, 1982). Dans le lit endigué de la Loire moyenne l'extension progressive de la ripisylve a été observée, comme sur le Rhône, depuis le milieu du XIX ${ }^{\mathrm{e}}$ siècle (Bazin et Gautier, 1996). Plusieurs facteurs contribuent à l'extension de la forêt en Loire moyenne : la séparation entre le lit endigué et le val, consécutive à la construction des levées, d'une part, a eu d'importantes conséquences sédimentaires et écologiques, notamment en termes d'incision du lit, aboutissant à l'extension de la ripisylve à travers la chenalisation progressive des écoulements et la déconnexion des annexes hydrauliques (Garcin et al., 2006). D'autre part le ralentissement récent de l'activité de la Loire, dû à l'absence de grandes crues, se traduit par une quasi-stabilité des méandres, avec pour conséquence une fermeture du lit par l'extension de la ripisylve (Leteinturier et al., 2000). Enfin, l'abandon progressif d'un grand nombre d'activités et d'usages sur le fleuve depuis la seconde moitié du XIX ${ }^{\mathrm{e}}$ siècle, est à l'origine d'une augmentation généralisée des surfaces occupées par les communautés forestières en Loire moyenne (Cornier, 2002). Le déclin quasi général des pratiques et des usages pastoraux dans le lit endigué de la Loire s'est accéléré dans la deuxième moitié du $\mathrm{Xx}^{\mathrm{e}}$ siècle, à partir de laquelle on assiste à l'émergence rapide d'une forêt alluviale presque continue, qui façonne le paysage actuel. Les coupes de végétation et le pâturage extensif sont les deux principales réponses préconisée par le Plan Loire Grandeur Nature à l'homogénéisation des espèces végétales liée à l'extension de la forêt (Burnouf et al., 2005).

Nous avons montré ici que cet espace endigué, revêtu d'une valeur écologique et patrimoniale associée à un caractère prétendument non domestiqué, « sauvage », est fortement structuré par les activités humaines et, malgré son caractère inondable, il est constitué d'un assemblage d'habitats semi-naturels et d'habitats très anthropisés, étroitement imbriqués.

Du point de vue méthodologique, la base de données du SIEL est intéressante pour l'information irremplaçable qu'elle délivre sur des habitats, comme les prairies inondables, mal connus à travers les bases de données de l'IGN, orientées vers les espaces urbanisés (BD Topo) ou boisés (BD Forêts) ou à travers le Référentiel Parcellaire Graphique, qui concerne les 
cultures majoritaires dans les îlots de cultures soumises à déclaration PAC. Il est donc regrettable que la mise à jour régulière du SIEL ait été abandonnée, la dernière couverture datant de 2005. L'utilisation de la classification issue du SIEL a montré les difficultés, pour les services de l'état, d'élaborer une classification détaillée nécessitant une reconnaissance fine de la végétation, ce qui explique l'affectation des polygones à des classes de rang varié. Une réorganisation de cette classification était donc inévitable pour faire de cette base de données une source d'information sur l'occupation du sol du lit endigué.

\section{Conclusion}

Les paysages fluviaux sont souvent considérés comme des espaces de nature, où les activités humaines interviendraient de façon uniquement indirecte. Dans le cas de la Loire cette position prend la forme du poncif « la Loire, dernier fleuve sauvage
d'Europe ». Bien que des travaux aient souligné le caractère « simpliste » (Rode, 2010a) de cette image, l'idée que le lit endigué de ce grand fleuve soit pauvre voire dépourvu d'activités anthropiques est tenace, et fonde une partie des travaux sur le patrimoine naturel ligérien. À travers l'exploration de la diversité de l'occupation du sol du lit endigué de la Loire moyenne, nous avons en revanche mis en évidence les usages et l'empreinte anthropique que traduisent certains habitats, comme les cultures, les espaces artificialisés et les diverses formations herbacées. Cela ne diminue en rien l'intérêt naturaliste de cet espace, d'autant plus que les activités humaines sont parfois à l'origine d'écosystèmes nouveaux non dépourvus d'intérêt écologique, comme souligné pour les espaces fluviaux du Rhône (Fruget et Michelot, 1997) et de l'Isère (Girel et Manneville, 1998; Girel, 2010). Cela oblige à penser les espaces fluviaux en étroite relation avec les activités agricoles et urbaines qui s’y déploient au cours du temps.

Annexe : Affectation des polygones du site d'étude : typologie du SIEL mêlant différents rangs (à gauche : libellé) et regroupement des classes utilisé ici (à droite : classe principale)

Polygons allocation in the study site: SIEL typology mixing different ranks (left) and merged classes (right)

\begin{tabular}{|c|c|c|c|}
\hline Code_typo & Libellé & $\begin{array}{l}\mathrm{N}^{\circ} \text { classe } \\
\text { principale }\end{array}$ & Classe principale \\
\hline 0100 & Sable & \multirow{2}{*}{1} & \multirow{2}{*}{ Sables } \\
\hline 0223 & Gravière & & \\
\hline 0200 & Eau & \multirow{5}{*}{2} & \multirow{5}{*}{ Eau courante et stagnante } \\
\hline 0210 & Eau courante & & \\
\hline 0220 & Eau stagnante & & \\
\hline 0221 & Plan d'eau naturel (Boire) & & \\
\hline 0222 & Plan d'eau artificiel (étangs, lacs de retenue) & & \\
\hline 1000 & Végétations aquatiques et semi-aquatiques & \multirow{11}{*}{3} & \multirow{11}{*}{$\begin{array}{l}\text { Végétation aquatique et semi-aqua- } \\
\text { tique }\end{array}$} \\
\hline 1100 & Communautés aquatiques & & \\
\hline 1110 & Peuplements algaux & & \\
\hline 1120 & Communautés à lentilles d'eau & & \\
\hline 1130 & Communautés à potamots & & \\
\hline 1200 & Communautés d'hélophytes, mégaphorbiaies hygrophiles & & \\
\hline 1220 & Phalaridaies & & \\
\hline 1230 & Magnocarièaies & & \\
\hline 1240 & Communautés d'amphiphytes & & \\
\hline 1250 & Autres communautés d'hélophytes & & \\
\hline 1261 & Herbier de Jussies & & \\
\hline 2000 & Végétations herbacées pionnières typiques du lit mineur & \multirow{5}{*}{4} & \multirow{5}{*}{ Végétation pionnière du lit mineur } \\
\hline 2100 & Communauté pionnière des vases humides (Nanocyperion) & & \\
\hline 2200 & Communauté pionnière des sables humides (Chenopodion rubri) & & \\
\hline 2300 & Communauté des basses vaseuses du lit mineur (Bidention tripartitae) & & \\
\hline 2500 & Végétations pionnières alluviales des sables secs du lit mineur & & \\
\hline
\end{tabular}


Francesca Di Pietro et al. - Norols n 242 (2017/1) p. 7-23

\begin{tabular}{|c|c|c|c|}
\hline 3100 & Communautés hygrophiles du lit majeur & \multirow{6}{*}{5} & \multirow{6}{*}{ Prairies du lit majeur } \\
\hline 3200 & Prairies humides atlantiques & & \\
\hline 3300 & Prairies mésophiles et mésoxérophiles du lit majeur & & \\
\hline 3310 & Prairies mésophiles pâturées du lit majeur & & \\
\hline 3320 & Prairie mésophile à avoine élevée & & \\
\hline 3330 & Prairie mésophile à mésoxérophile à chiendents dominants & & \\
\hline 3500 & Pelouses et autres communautés xérophiles à mésophiles du lit majeur & \multirow{6}{*}{6} & \multirow{6}{*}{ Pelouses et friches } \\
\hline 3510 & Pelouse à Sedum sp. plur. (orpins) dominants & & \\
\hline 3540 & Pelouses, landes à Artemisia campestris L. (Armoise champêtre) & & \\
\hline 3550 & Autres friches herbacées & & \\
\hline 3600 & Landes à genêts & & \\
\hline 3700 & Fruticées & & \\
\hline 4100 & Forêt de bois tendres & \multirow{10}{*}{7} & \multirow{10}{*}{ Forêts de bois tendre } \\
\hline 4110 & Saulaie arbustive & & \\
\hline 4111 & $\begin{array}{l}\text { Saulaie arbustive à Salix triandra L. emend. Ser. (Saule à trois étamines) et } \\
\text { à Salix viminalis }\end{array}$ & & \\
\hline 4120 & Saulaie peupleraie arbustive & & \\
\hline 4130 & Saulaie à Salix alba L. (Saule blanc) & & \\
\hline 4140 & Saulaie-peupleraie arborescente & & \\
\hline 4141 & Saulaie-peupleraie à Populus nigra L. (peuplier noir) & & \\
\hline 4150 & Forêt de bois tendres colonisés par les bois durs & & \\
\hline 4151 & Peupleraie sèche & & \\
\hline 4160 & Peuplements de substitution à grandes renouées exotiques & & \\
\hline 4200 & Forêt de bois durs & \multirow{13}{*}{8} & \multirow{13}{*}{ Autres forêts } \\
\hline 4210 & Frênaie, ormaie à Fraximus et Ulmus & & \\
\hline 4211 & Frênaie-ormaie typique & & \\
\hline 4212 & Frênaie fraîche & & \\
\hline 4214 & Haie bocagère mixte & & \\
\hline 4220 & Chênaie alluviale à Quercus robur L. (Chêne pédonculé) & & \\
\hline 4230 & Chênaie planitiaire ou collinéenne à Quercus robur L. (Chêne pédonculé) & & \\
\hline 5000 & Autres forêts et boisements & & \\
\hline 5100 & Forêts naturelles & & \\
\hline 5200 & Robineraie (plantation de Robinier faux-acacia) & & \\
\hline 5300 & Arbres d'alignement & & \\
\hline 5400 & Peupleraie plantée & & \\
\hline 5500 & Autres plantations d'arbres & & \\
\hline 6000 & Cultures & \multirow{3}{*}{9} & \multirow{3}{*}{ Cultures } \\
\hline 6400 & Grandes cultures & & \\
\hline 6500 & Autres cultures & & \\
\hline 6100 & Vergers & \multirow{3}{*}{10} & \multirow{3}{*}{ Vignes et vergers } \\
\hline 6200 & Jardins & & \\
\hline 6300 & Vignes & & \\
\hline 6600 & Surfaces agricoles non cultivées & \multirow{3}{*}{11} & \multirow{3}{*}{ Jachères } \\
\hline 6610 & Jachères & & \\
\hline 6620 & Friches agricoles & & \\
\hline 9999 & Espaces anthropisés & 12 & Espace artificialisé \\
\hline
\end{tabular}




\section{Bibliographie}

Barraud R. et al., 2013. Les épis de la Loire armoricaine, un héritage à la patrimonialité incertaine, Norois, 228(3), p. 39-52.

Barraud R., 2011 . Rivières du futur, wild rivers ?, Vertigo, 10 (décembre, mis en ligne le 30 novembre 2011).

Bazin P., Gautier E., 1996. Un espace de liberté pour la Loire et l'Allier : de la détermination géomorphologique à la gestion. Revue de géographie de Lyon, 71(4), p. 377-386.

Bonin S., 2007. Fleuves en ville : enjeux écologiques et projets urbains, Strates. Matériaux pour la recherche en sciences sociales, 13 (mis en ligne le 22 octobre 2008).

Bonin S., 2001. Paysages et représentations dans les guides touristiques. La Loire dans la collection des Guides-Joanne, Guides Bleus (1856 à nos jours), L'espace géographique, 30, p. $111-126$.

TER BraAk C.J.F., Wiertz J., 1994. On the statistical analysis of vegetation change: a wetland affected by water extraction and soil acidification, Journal of Vegetation Science, 5 (1983), p. 361-372.

Burnouf J. et al., 2005. Une autre Loire : dynamiques socioenvironnementales sur 15000 ans, Covalences, (56), p. 4-9.

Charrier M.P., 2000. Entre Anjou et Pays Nantais, interventions humaines et transformations hydro-morphologiques en Loire armoricaine (1750-1960), Annales de Géographie, 109 (612), p. 115-131.

Cornier T., 2002. La végétation alluviale de la Loire entre le Charolais et l'Anjou : essai de modélisation de l'hydrosystème, thèse de doctorat en écologie végétale, Université François Rabelais Tours, 227 p.

Cyprien A.L., Visset L., Carcaud N., 2004. Evolution of vegetation landscapes during the Holocene in the central and downstream Loire basin (Western France), Vegetation History and Archaeobotany, 13, p. 181-196.

DIREN Centre A., 1994. Guide de lecture des cartes de végétation du lit de la Loire et de ses affluents, SIEL (Système d'Information sur l'Évolution du Lit de la Loire et de ses affluents) - cartes de végétation.

Féménias D., Sirost O., 2011 . Fleuves, estuaires, cours d'eau : représentations et pratiques, Vertigo, 10 (Décembre 2011, mis en ligne le 30 novembre 2011).

Fernandes M.R., Aguiar F.C., Ferreira M.T., 2011 . Assessing riparian vegetation structure and the influence of land use using landscape metrics and geostatistical tools, Landscape and Urban Planning, 99 (2), p. 166-177.

Folléa X., Gautier Y., 2009. Atlas des paysages du Loir-etCher, CAUE Loir-et-Cher, DIREN Centre.

Freeman R.E., Ray R.O., 2001. Landscape ecology practice by small scale river conservation groups, Landscape and Urban Planning, 56(3-4), p.. 171-184.

Fruget J., Dessaix J., 2003. Changements environnementaux, dérives biologiques et perspectives de restauration du Rhône français après 200 ans d'influences anthropiques, Vertigo, 4 (3, mis en ligne le $1^{\text {er }}$ décembre 2003).

Garcin M. et al., 2006. Impacts des héritages sur un hydrosystème : l'exemple des levées en Loire moyenne et océanique, in Allée P., Lespez L. (dir.), L'érosion entre société, climat et paléoenvironnement, Clermont-Ferrand, Presses universitaires Blaise-Pascal, p. 225-236.

Gatien A., Corbonnois J., Laurent F., 2009. Une analyse de paysages comme préalable à l'étude des systèmes agraires : application à la vallée du Loir, Norois, 213(4), p. 89-101.

Gatien A., Laurent F., Corbonnois J., 2011 . Pratiques agricoles et ressources en eau des vallées principales du bassin versant de la Maine : quelles représentations?, Vertigo, 10 (décembre 2011, mis en ligne le 30 novembre 2011).

Germaine M.-A., 2008. Évaluer la diversité des paysages de vallées. Réalisation d'une typologie en Basse-Normandie, Norois, 207(2), p. 7-19.

Germaine M.-A., Barraud R., 2013. Restauration écologique et processus de patrimonialisation des rivières dans l'Ouest de la France, Vertigo, 16 (juin 2013, mis en ligne le 30 mai 2013).

Girel J., 2010. Histoire de l'endiguement de l'Isère en Savoie : conséquences sur l'organisation du paysage et la biodiversité actuelle, Géocarrefour, 85(1), p. 41-54.

Gordon L.J., Peterson G.D., Bennett E.M., 2008. Agricultural modifications of hydrological flows create ecological surprises, TRENDS in Ecology and Evolution, 23(4), p. 211 219.

Grivel S., Gautier E., 2012. Mise en place des îles fluviales en Loire moyenne, du XIX ${ }^{\mathrm{e}}$ siècle à aujourd'hui, Cybergeo, doc 615 (mis en ligne le 24 juillet 2012).

Gueydon F., Maillet G., 2010. La répartition des cultures dans la zone inondable de la Loire entre Saumur et SaintFlorent-le-Vieil, Cahiers nantais, 1 et 2, p. 13-25.

Harrison P.A. et al., 2010. Identifying and prioritising services in European terrestrial and freshwater ecosystems, Biodiversity and Conservation, 19(10), p. 2791-2821.

Joliet F., Beaujouan V., Jaсов M., 2004. Quelle naturalité du paysage ligérien? La Loire du Maine-et-Loire, Norois, 192(3), p. 85-94.

LECEUR Y., 2011. La Loire moyenne naviguée au XIX ${ }^{\mathrm{e}}$ siècle : représentations, réglementation et aménagement, Vertigo, Hors-série (décembre 2011, mis en ligne le 30 novembre 2011).

Leps J., Smilauer P., 2003. Multivariate Analysis of Ecological Data using CANOCO, Cambridge University Press.

Lespez L., Ballouche A., 2009. Paysages au fil de l'eau : l'objet, le sensible et leurs trajectoires, Bulletin de l'association des géographes français, 1, p. 3-11.

Leteinturier B. et al., 2000. Morphodynamisme d'un tronçon de Loire bourbonnaise depuis le XVIII siècle, Géomorphologie : relief, processus, environnement, 6(4), p. 239-252.

LÉvÊque C., 2011 . Des fleuves et des estuaires : Pour qui ? Pour quoi ? L'émergence de l'interdisciplinarité dans l'étude des hydrosystèmes. Vertigo, 10 (décembre 2011, mis en ligne le 30 novembre 2011).

Loire-Bretagne, S.D.B., 2002. Restauration et entretien du lit de la Loire et de ses affluents,

VAN Looy K. et al., 2014. Integrated modelling of functional and structural connectivity of river corridors for European otter recovery, Ecological Modelling, 273, p. 228-235. 
VAn Looy K., Meire P., 2009. A conservation paradox for riparian habitats and river corridor species, Journal for Nature Conservation, 17(1), p. 33-46.

Van Looy K., Meire P., Wasson J.G., 2008. Including riparian vegetation in the definition of morphologic reference conditions for large rivers: A case study for Europe's Western Plains, Environmental Management, 41, p. 625-639.

Montembault D., 2004. L'histoire comparée du Val d'Authion et de la Loire armoricaine en Anjou Pour comprendre la nouvelle appropriation citadine des paysages ligériens, Norois, 192(3), p. 47-62.

Pautou G., Bravard J.-P., 1982. L'incidence des activités humaines sur la dynamique de l'eau et l'évolution de la végétation dans la vallée du Haut-Rhône français, Revue de géographie de Lyon, 57(1), p. 63-79.

Pelletier J., 1990. Sur les relations de la ville et des cours d'eau, Revue de géographie de Lyon, 65(4), p. 233-239.

Pin B., Rode S., Servain S., 2013. Processus de construction d'une ressource territoriale valorisant le patrimoine naturel et culturel en Loire tourangelle, Vertigo, 16 (juin 2013), mis en ligne le 30 mai 2013.

Pradeau C., 1999. Réseaux hydrographiques et organisation de l'espace, L'Information géographique, 63, p. 3-9.

Rode S., 2010a. De l'aménagement au ménagement des cours d'eau : le bassin de la Loire, miroir de l'évolution des rap- ports entre aménagement fluvial et environnement. Cybergeo, document 5 (mis en ligne le 22 septembre 2010).

Rode S., 2010b. Reconquête urbaine de la Loire et risque d'inondation : des représentations aux aménagements urbains, Géocarrefour, 85(3), p. 221-228.

RolLin J., 2010. La protection des petites rivières périurbaines dans les Bouches-du- Rhône depuis les années 1960 : une analyse de la construction locale de la norme environnementale, Géocarrefour, 85(3), mis en ligne le 15 mars 2011.

Romain F., 2014. L'imaginaire fluvial contemporain : un antidote urbain ? Le cas du Lez vert à Montpellier et de la Têt à Perpignan. Projets de paysage, Publié le 27 septembre 2014.

Romain F., 2010. Le fleuve, porteur d'images urbaines: formes et enjeux, Géocarrefour, 85(3), mis en ligne le 15 mars 2011.

Schaich H., Rudner M., Konold W., 2010. Short-term impact of river restoration and grazing on floodplain vegetation in Luxembourg, Agriculture, Ecosystems and Environment, 139(1-2), p. 142-149.

Tricart J., Bravard J.-P., 1991. L'aménagement des trois plus grands fleuves européens: Rhin, Rhône et Danube. Problèmes et méfaits, Annales de Géographie, 100(561), p. 668-713.

ULTSCH J., 2010. Les temporalités de l'interface ville- rivière à travers le cas de Saint-Étienne et du Furan, Géocarrefour, 85(3), mis en ligne le 15 mars 2011. 